\section{Dental Journal}

\author{
M(ajalah Kedokteran Gigi
}

Research Report

\section{Dental Journal \\ (Majalah Kedokteran Gigi) \\ 2016 September; 49(3): 158-162}

\title{
Inhibitory effects of siwak (Salvadora persica. L) extract on the growth of Enterococcus faecalis planktonics and biofilms in vitro
}

\author{
Ika Rhisty Cendana Sari, ${ }^{1}$ Rini Devijanti Ridwan, ${ }^{2}$ and Diah Savitri Ernawati ${ }^{3}$ \\ ${ }^{1}$ Badan Penyelenggara Jaminan Sosial (BPJS) Kesehatan, Malang-Indonesia \\ ${ }^{2}$ Department of Biology Oral, Faculty of Dental Medicine, Universitas Airlangga, Surabaya - Indonesia \\ ${ }^{3}$ Department of Oral Medicine, Faculty of Dental Medicine, Universitas Airlangga, Surabaya - Indonesia
}

\begin{abstract}
Background: Enterococcus faecalis (E. faecalis) is one of the most persistent gram positive bacteria in root canal, resulting in secondary infection after endodontic treatment. E. faecalis pathogenicity is caused by overgrowth of E. faecalis planktonics and biofilms. E. faecalis planktonics produce lipoteichoid acid (LTA) as a virulence factor that can defend their permeability cell. On the other hand, E. faecalis biofilms produce protease, such as Esp (enterococcal surface protein), GelE (gelatinase), and SprE (serin protease), that have quorum-sensing mechanism as an adhesion factor to form extracellular polysaccharide substance (EPS) and increase the growth of the biofilms themselves. Siwak (Salvadora persica L.) has active components, namely benzylisothio-cyanate, trimethylamine, and salvadorine that can inhibit the growth of E. faecalis planktonics and biofilms. Purpose: This study aimed to measure inhibitory effects of siwak extract on the growth of E. faecalis planktonics and biofilms. Method: This research was an antimicrobial research on the culture of E. faecalis incubated in a TSB medium. Siwak extract was diluted into different concentrations, namely 25\%, 30\%, 35\%, $40 \%, 45 \%, 50 \%, 55 \%, 60 \%, 65 \%, 70 \%, 75 \%$, and 100\%. The extract then was placed into the E. faecalis's colony and planted into Trypticase Soy Agar medium. After incubated for 24 hours at $37^{\circ} \mathrm{C}$, the colony would be measured and compared with the control (+) and control (-). As an antibiofilm research, this research used biofilm microtitter assay method to form E. faecalis biofilms incubated in a well-plate medium containing TSB and $0.1 \%$ glucose. Siwak extract then was diluted into the same range concentration as in first method, and placed into the colony of E. faecalis to form biofilms. The biofilms were measured and compared to the control (+) given siwak extract and the control (-) given $0.1 \%$ chlorhexidine. After the incubation, they were washed three times, and staining process was conducted using Chrystal violet. The optical density then was measured by ELISA Reader $595 \mathrm{~nm}$. Result: Siwak extract could inhibit the growth of E. faecalis planktonics at the concentration of $35 \%$ as a minimum inhibitory concentration as well as the growth of E. faecalis biofilms at the concentration of $45 \%$ as a minimum biofilm inhibitory concentration. Conclusion: Siwak extract has an inhibitory effect, particularly at a concentration of $35 \%$ on the growth of E. faecalis planktonics and at the concentration of $45 \%$ on the growth of E. faecalis biofilms.
\end{abstract}

Keywords: Enterococcus faecalis; Siwak; antibacterial; antibiofilm; benzylisothio-cyanate; trimethylamine; salvadorine 


\section{INTRODUCTION}

Enterococcus faecalis (E. faecalis) is one of the most resistant gram-positive bacteria in root canal. E. faecalis can cause a secondary infection of the root canal after endodontic treatment. E. faecalis bacterial pathogenicity can be triggered by the growth of E. faecalis planktonics and biofilms. ${ }^{1}$

One of the herbs currently widely used by society in maintaining their oral health is siwak rod (Salvadora persica.L). Siwak is known to have a wide variety of useful components. Trimethylamine and benzyl-isothiocyanate in siwak serve to reduce the occurrence of adhesion to the tooth surface and inhibit the accumulation of plaque. Antimicrobial and cleaning effects of Siwak have been indicated by chemical content variations, such as high benzyl-isothiocyanate, specific alkaloid salvadorine, trimethylamine, saponins, flavonoids, tannins, vitamin $C$, plant sterols, silica, sodium chloride, resins, and potassium chloride (salvadourea). ${ }^{2}$

Moreover, siwak extract with distilled solvent, based on a previous research conducted by Abdelrahman, can inhibit the growth of Streptococcus mutans and Streptococcus faecalis at a concentration of $50 \%$. Another previous research conducted by Almas also shows that siwak extract with ethanol at a concentration of $25 \%$ can lead to the absence of the bacterial growth of S. mutans and Streptococcus sanguinis. ${ }^{3,4}$ Those bacteria are Gram-positive bacteria commonly found in endhodontic infections. ${ }^{5}$ Those results of the previous researches underlie the objective of this research to measure the effectiveness of siwak extract in inhibiting the growth of E. faecalis biofilms since siwak extract contains potential substances that can be used as an irrigation and antiseptic material. In other words, this research aimed to reveal whether siwak extract (Salvadora persica.L) could inhibit the growth of E. faecalis and its biofilms by in vitro.

\section{MATERIALS AND METHOD}

This research was an experimental study with true experiment-post test only control group design. This research was conducted at the Laboratory of RSPTI (Hospital of Tropical and Infectious Diseases) in Surabaya form June 2014 to October 2015. The research samples used for the inhibition test of the growth of bacteria were $E$. faecalis bacterial cultures obtained from the Laboratory of Hospital of Tropical and Infectious Diseases in Surabaya. Inoculum of each bacterium was taken from the cultures, incubated on trypticase soy broth at $37^{\circ} \mathrm{C}$ temperature for 18 hours, and then diluted with $0.85 \%$ sterile $\mathrm{NaCl}$ solution (Sigma-Aldrich Inc., St. Louis, MO, USA) to achieve suspension turbidity equivalent to $0.5 \mathrm{Mc}$.Farland Standard. ${ }^{1,5}$

In addition, the research samples used for the inhibition test of the growth of bacteria were E. faecalis bacterial cultures that could form biofilms indicated through 100 $\mu \mathrm{L}$ of cultured bacteria suspension in polyprophylene tube containing $2 \mathrm{~mL}$ of trypticase soy broth (TSB) (Merck Milipore Inc., Darmstadt, Germany) with the addition of $1 \%$ glucose for 48 hours at a temperature of $37^{\circ} \mathrm{C}$. After 48 hours of the incubation, biofilm cells were harvested by removing the culture medium, and then the tube was rinsed three times with $200 \mu \mathrm{L}$ of phosphate buffer saline (pH 7.2) (Sigma-Aldrich Inc., St. Louis, MO, USA) to remove non-adhesive part of the bacteria. Meanwhile, the adhesive part of the bacteria was harvested through vortex and centrifugation processes. The pellets then were suspended in PBS to be conditioned in 0.5 McFarland turbidity standard (cloudiness). ${ }^{1}$ The number of samples in each type of treatment, moreover, was four (4) samples. Those samples in each type of treatment were treated using the siwak extract through sokhletation process at the concentrations of $100 \%(1 \mathrm{~g} / \mathrm{ml}), 75 \%(0.75 \mathrm{~g} / \mathrm{ml}), 70 \%$ $(0.7 \mathrm{~g} / \mathrm{ml}), 65 \%(0.65 \mathrm{~g} / \mathrm{ml}), 60 \%(0.6 \mathrm{~g} / \mathrm{ml}), 55 \%(0.55$ $\mathrm{g} / \mathrm{ml}), 50 \%(0.5 \mathrm{~g} / \mathrm{ml}), 45 \%(0.45 \mathrm{~g} / \mathrm{ml}), 40 \%(0.4 \mathrm{~g} /$ $\mathrm{ml}), 35 \%(0.35 \mathrm{~g} / \mathrm{ml}), 30 \%(0.3 \mathrm{~g} / \mathrm{ml})$, and $25 \%(0.25 \mathrm{~g} /$ $\mathrm{ml})$ obtained through dilution method.

$10 \mathrm{ml}$ of the siwak extract was planted on each culture in a petri dish using a micropipette, and then incubated on solid Mueller Hinton media (bioMerieux Industry, Inc., Philadelphia, PA, USA) for $24 \mathrm{~h}$ at $37^{\circ} \mathrm{C}$. The number of colonies then was counted using a colony counter. To measure inhibitory effects on the bacterial biofilm growth, each well containing $100 \mu \mathrm{L}$ of TSB $+1 \%$ glucose was added with $100 \mu \mathrm{l}$ of $E$. faecalis bacterial cultures proven to produce biofilms. $100 \mu \mathrm{l}$ of the siwak extract then was added into the wells of each treatment, and incubated for 48 hours at $37^{\circ} \mathrm{C}$. After that the well-plates were placed in an anaerobic jar for 24 hours. The wells then were washed $3 \mathrm{x}$ with $0.2 \mathrm{ml}$ of PBS, and then staining process was conducted using $0.2 \mathrm{ml}$ of crystal violet. They were rinsed again with distilled water, and then added with $0.2 \mathrm{~mL}$ of sterile acidified isopropanolol (Honeywell International Inc., Morristown, NJ, USA). ${ }^{1}$ Optical Density (OD) values in the wells of each treatment were read using ELISA Reader with a wavelength of $595 \mathrm{~nm}$ (Bio-Rad Laboratories, Inc., Berkeley, CA, USA). ${ }^{1}$

\section{RESULTS}

The results showed that the growth of the bacteria declined as the increasing of the concentration of the siwak extract used. The growth of E. faecalis bacteria was detected after the provision of the siwak extract at the concentrations of $25 \%$ to $35 \%$. Meanwhile, there was no bacterial colony grown after the provision of the siwak extract at the concentration of $40 \%$. Furthermore, there was also no bacterial colony grown after the provision of the siwak extract at the concentrations of $45 \%$ to $100 \%$.

The results of the Anova test, furthermore, indicated that there were significant differences between the research 
Table 1. Results of LSD test on bacterial inhibitory effects

\begin{tabular}{ccc}
\hline Concentration (\%) & Number of Samples & SD \\
\hline 25 & 4 & 102.956 \\
30 & 4 & 0.68981 \\
35 & 4 & 0.76158 \\
40 & 4 & $.00000^{\mathrm{c}}$ \\
45 & 4 & $.00000^{\mathrm{c}}$ \\
50 & 4 & $.00000^{\mathrm{c}}$ \\
55 & 4 & $.00000^{\mathrm{c}}$ \\
60 & 4 & $.00000^{\mathrm{c}}$ \\
65 & 4 & $.00000^{\mathrm{c}}$ \\
70 & 4 & $.00000^{\mathrm{c}}$ \\
75 & 4 & $.00000^{\mathrm{c}}$ \\
100 & 4 & $.00000^{\mathrm{c}}$ \\
Control (+) & 4 & 373.363 \\
Control (-) & 4 & $.00000^{\mathrm{c}}$ \\
\hline
\end{tabular}

Table 2. Results of LSD test on bacterial biofilm inhibitory effects

\begin{tabular}{ccc}
\hline Concentration (\%) & Number of Samples & SD \\
\hline 25 & 4 & 0.0133 \\
30 & 4 & 0.01794 \\
35 & 4 & 0.03244 \\
40 & 4 & 0.0466 \\
45 & 4 & 0.01117 \\
50 & 4 & 0.02193 \\
55 & 4 & 0.01439 \\
60 & 4 & 0.03063 \\
65 & 4 & 0.01192 \\
70 & 4 & 0.022411 \\
75 & 4 & 0.0229 \\
100 & 4 & 0.00569 \\
Control (-) & 4 & 0.02723 \\
(chlorhexidine) & & \\
Control (+) & 4 & 0.11877 \\
medium + bacteria) & & 0.0637 \\
Extract Control & 4 & \\
(only extract) & & \\
Staining Control & 4 & \\
(only medium) & &
\end{tabular}

groups ( $\mathrm{p}$ value $<0.05$ ). Thus, Least Square Differences (LSD) test was performed. The results of the LSD test demonstrated that there was no significant difference between the provision of the extract at the concentration of $35 \%$ and that at the higher concentration. Therefore, the siwak extract at the concentration of $35 \%$ was considered as the minimum inhibitory concentration against the growth of E. faecalis bacteria.
In addition, the results of the optical density readings indicated that the growth of bacterial biofilms tended to decrease, in line with the increasing of the concentration of the siwak extract used, characterized by the decreased values of optical density and the adhesive cell attachment at the less visible staining.

The results of the LSD test, moreover, showed that there was no significant difference between the provision of the siwak extract at the concentration of $25 \%$ and at the concentration of $40 \%$. There was a significant difference between the provision of the siwak extract at the concentration of $45 \%$ and at the lower concentration.

The concentration of $45 \%$, as a result, can be considered as the minimum inhibitory concentration for biofilms. Further analysis found that there was no significant difference between the concentration of $45 \%$ and the higher ones, namely $50 \%, 55 \%, 60 \%, 65 \%, 70 \%$, and $75 \%$. In other words, the lowest concentration of the siwak extract to inhibit the growth of biofims was $45 \%$. However, siwak extract at the concentration of $100 \%$ was not significantly different from the extract at the concentrations of $50 \%$ to $75 \%$.

\section{DISCUSSION}

The growth of E. faecalis bacteria can continually occurs by maintaining the resistance of the bacterial cell wall and cell membrane in the presence of lipoteichoid acid (LTA), capable of performing defense of external bacterial membrane permeability and peptidoglycan playing a role in maintaining the cell shape. ${ }^{6}$ A previous research even shows that siwak extract with distilled solvent can inhibit the growth of $S$. mutans and $S$. faecalis bacteria from a low concentration to a concentration of $50 \% .^{3,4}$ Another in vitro research shows that the use of ethanol in siwak extract at a concentration of $25 \%$ triggers no growth of $S$. mutans and $S$. sanguinis bacteria classified as cocci-shaped gram-positive bacteria, similar to E. faecalis, involved in endodontic infections. Consequently, this research used the same material extract. ${ }^{3,4}$

In this research, ethanol at a concentration of $35 \%$ used in the siwak extract significantly inhibited the growth of E. faecalis bacteria. The concentration of $35 \%$ could be considered as the minimum inhibitory concentration. It means that siwak extract had an anti-bacterial ability, almost similar to the result of the previous research, but it requires a slightly higher concentration to inhibit $E$. faecalis bacteria than to inhibit other gram-positive bacteria. In other words, siwak, extracted using alcohol solvent, is an effective antibacterial material to inhibit $E$. faecalis bacteria. ${ }^{4,7}$

This inhibitory effects on the growth of E. faecalis bacteria indicate that there are disturbances in mechanisms of peptidoglycan and lipoteichoic acid (LTA) as well as metabolism and virulence of E. faecalis. Siwak extract is considered as an effective anti-bacterial material due to benzylisothio-cyanate, salvadorine, and trimethylamine 
contained. ${ }^{8}$ A previous research using mass spectroscopy shows that benzylisothio-cyanate is the largest component contained in the siwak extract. Benzylisothio-cyanate can trigger oxidase process, thus inhibiting the mechanism of bacterial metabolism. Meanwhile, salvadorine as one of specific alkaloids in siwak can inhibit both activity of enzymes produced by bacteria in synthesizing protein and synthesis of cell walls, interfere metabolic processes of bacteria, as well as produce organic anionic group resulting in unstable bacterial cell membrane leading to lysis and cell death. ${ }^{8}$

The mechanism of siwak extract in inhibiting the growth of $E$. faecalis bacteria correlates with a previous research showing that $17 \%$ EDTA, $\mathrm{CaOH}_{2}$, and $0.2 \%$ chlorhexidine also have an anti-bacterial ability against $E$. faecalis. 17\% EDTA alter cell membrane permeability, thus destroying the bacterial cell wall. Chlorhexidine works by damaging the cytoplasmic membrane of bacterial cells so the intracellular matrix secreted from the cells, leading to lysis. ${ }^{9} \mathrm{CaOH}_{2}$ can also be considered as an antibacterial material due to its ability to damage LTA, known as a major virulence factor in gram-positive bacteria. In other words, the mechanism of siwak extract is similar to the endodontic treatment material that inhibits the growth of E. faecalis bacteria. ${ }^{8}$

The growth of E. faecalis is known to generate biofilms. Biofilms can form and grow due to adhesion factors and aggregation substance, such as exopolysaccharide, as well as a variety of biofilm associated protein. One of proteins associated to the biofilm formation is Esp which acts as a specific protein in inducing bacterial colonization and bacterial colony attachment on the surface of the substrate. Esp together with other specific proteins, such as serine protease (SprE) can initiate E. faecalis biofilm formation, both in vitro and in vivo. The process of $E$. faecalis biofilm formation is also triggered by both ace (collagen binding antigen) that has strong adhesion to dentin, as well as GelE that plays a role in the process of bacterial quorum-sensing and induces extracellular matrix as a response to biofilm formation. ${ }^{10}$

The whole of virulence factors that are able to form extracellular polymeric substances (EPS) are attached to the bacteria themselves and correlated to the formation of bacterial biofilms. Adhesions occur in stages. The first stage occurs in a reversible way, then becoming into permanent (irreversible) and undergoing maturation and aggregation to form biofilms. Biofilm formation itself is not essential to trigger pathogen infection, but increased bacterial resistance in bacteria when forming biofilms can trigger virulence factor in bacteria to work maximally, leading to increased pathogenicity. ${ }^{6}$

This EPS formation process is influenced by signal transduction mechanisms, called as quorum-sensing. Bacterial adhesions occur in the surface of the matrix, in which bacteria form stable microcolonies and emit chemical signals as communication between cells. When communication between these cells reach threshold intensity level, EPS production will be activated so that the attachment of microcolonies multiplies and thickens resulting in the attachment of macrocolonies to the extracellular matrix. In other words, the thicker the layer forming biofilms is, the lower the nutrient and $\mathrm{pH}$ conditions in the surface of the matrix is, encouraging the release of a colony form the outermost layer of the matrix to alter again into bacterial planktonics. ${ }^{11,12}$ With the increasing of planktonic cells due to the release of the colony, the virulence and pathogenicity of E. faecalis bacteria will increase. ${ }^{13,14}$

In the second previous research, siwak extract is tested on E. faecalis biofilms. The main elements contain in siwak extract, such as benzylisothio-cyanate, trimethylamine, and salvadorine, also have effectiveness against bacterial biofilms. Trimethylamine in siwak can inhibit the adhesion factor on the surface of the substrate thereby inhibiting the formation of bacterial biofilms. Benzylisothio-cyanate, on the other hand, can react to sulfhydryl groups contained in the protein enzyme associated to biofilm formation, leading to cell death and biofilm-forming inhibition. Benzylisothiocyanate in siwak has led to anti-quorum sensing capability of the enzyme protein produced by E. faecalis. ${ }^{6,15}$

In another previous research on E.faecalis biofilms, microtiter biofilm assay method is used to see the effects of fsr gene on the activation of gelatinase-serine protease (Gele-SPRE) operon expressions. The mechanism of GelE-sprE operon also becomes quorum-sensing in the formation of E. faecalis biofilms. Thus, it can be concluded that the result of this previous research indicates that this method are relevant to the activities of specific genes in the formation and growth of $E$. faecalis biofilms resulting in tissue destruction causing endodontic infection. ${ }^{13,14}$

By using microtiter assay method, the optical density (OD) values of the bacterial biofilms obtained was read by ELISA reader with a wavelength of $595 \mathrm{~nm}$. OD values measured based on turbidity colors absorbed by the biofilms have several complicating factors, such as turbidity of extracts used that can affect the thickness of the biofilms contained. ${ }^{6}$ Consequently, in this research staining and turbidity were controlled, so the staining and turbidity of siwak extract did not significantly affect. Thus, in this research the smaller the concentration of siwak extract was used, the higher the OD values were. In other words, the larger the OD values were obtained, the thicker the biofilms were generated.

The thicker biofilms indicates the greater resistance to anti-bacterial material. It means that anti-bacterial material will be more difficult to penetrate into the biofilms. As a result, the growth and formation of the biofilms will be more difficult to inhibit. Bacteria that can form biofilms have a slowdown in their metabolic rate and bacterial planktonic colony growth. ${ }^{6}$

This slow metabolic rate contributes to bacterial resistance to antibiotics, one of which is B-lactam. Antibiotics work by targeting growth factors working during the high bacterial growth, so they become not optimum and resistant to antibiotics. ${ }^{6}$ Similarly, the 
results of this research showed that siwak extract as an alternative for anti-biofilm material that could disturb the mechanism of bacterial resistance and inhibit the growth of the bacteria. Therefore, it can be said that siwak extract has effectiveness as anti-biofilm agent against $E$. faecalis bacteria at a minimum concentration of $45 \%$.

Nevertheless, it took a greater concentration to inhibit the growth of E. faecalis biofilms than to inhibit the growth of E. faecalis planctonics in this research. Similarly, previous researches and literatures also show that $E$. faecalis biofilms are more resistant to antibacterial than $E$. faecalis planctonics. Bacterial biofilms reported to have a resistance of 10 to 1000-fold to antibiotics in the process of phagocytosis compared to bacterial planktonics. ${ }^{16}$

To compare to another antimicrobial agent frequently used as a root canal irrigation to reduce the growth of bacterial biofilms, chlorhexidine at a concentration of $0.1 \%$ was used. According to a previous research, chlorhexidine at the concentration of $0.1 \%$ is effective to suppress the number of aerobic and anaerobic bacteria in oral cavity up to $97 \%$. However, chlorhexidine at the concentration of $0.1 \%$ is more effective against Gram-positive bacteria than against Gram-negative bacteria. Chlorhexidine at the concentration of 0.1 to $2 \%$, moreover, is also considered as anti-bacteriostastic material, but its activities depend on a condition of $\mathrm{pH}$. Its activities also trigger a damage to cells forming periodontal ligament tissue, oral mucosal irritation, burning sensation, and changes in taste perception. ${ }^{17}$

On the other side, a previous research conducted by Sukkarwalla et al. ${ }^{4}$ reports that siwak does not destruct periodontal tissues, and has a better tolerance level with both changes in the perception of taste and burning sensation. For those reasons, chlorhexidine at the lowest concentration was used in this research since it was expected to be effective as an antimicrobial in this antibiofilm test, ie $0.1 \%$. Based on the analysis of data, the use of siwak extract at the concentrations of $70 \%, 75 \%$, and $100 \%$ did not have significant differences from the use of chlorhexidine at the concentration of $0.1 \%$ (Table 2). As a result, it can be said that siwak extract has an ability to inhibit the growth of the biofilm with the level of effectiveness almost equal to chlorhexidine. ${ }^{17}$

Besides compared to the root canal irrigation material, siwak was also compared to another root canal treatment material since it has antibacterial and antibiofilm activities. In a previous research conducted by Zhang, ${ }^{9}$ cetrimide at a concentration of $0.2 \%$ reduce adhesion factor that can inhibit the formation and growth of bacterial biofilms. Cetrimide at the concentration of $0.2 \%$ with the ability to reduce the adhesion factor also can allow for the attachment of bacterial colonies apart so that the number of bacterial planktonics increase.

Similarly, trimethylamine contained in siwak extract can inhibit bacterial adhesion factors. Other elements contained in siwak extract, such as benzylisothio jointcyanate and salvadorine, are also capable of inhibiting the formation of acids and enzymes as products of bacterial metabolism, initiating the formation and defense of bacteria cell wall. With those capabilities, siwak extract can reduce the growth of bacterial planktonics and biofilms. Therefore, siwak extract can be used as an alternative to root canal treatment since it is not only safe for periodontal tissue, either as a root canal irrigation material or as a root canal sterilization material, but also effective to inhibit the growth of E. faecalis bacteria often found in cases of endhodontics. ${ }^{9}$ Finally, it can be concluded that the active components of siwak can inhibit the growth of E. faecalis bacteria, both E. faecalis planktonics and biofilms. Siwak can also be considered as an antibiofilm agent derived from natural materials.

\section{REFERENCES}

1. Oli AK, Raju S, Rajeshwari, Nagaveni S, Kelmani C. Biofilm formation by multidrug resistant Enterococcus faecalis (MDEF) originated from clinical samples. Journal Microbiol Biotech Res 2012; 2(2): 284-8.

2. Halawany HS. A review on miswak (salvadora persica) and it's effect on various aspects of oral health. The Saudi Dental Journal 2012; 24: 63-9.

3. Haque M. A review of the therapeutic effects of using miswak (salvador persica) on oral health. Saudi MEdical Journal 2015; 36(5): 530-43.

4. Sukkarwalla A, Salima M, Pranee L, Farzeen T. Efficacy of miswak on oral pathogens. Dent Res J (Isfahan) 2013; 10(3): 314-20.

5. Masadeh MM, Shadi G, Karem A. Antimicrobial activity of common mouthwash solutions on multidrug-resistance bacterial biofilms. J Clin Med Res 2013; 5(5): 389-94.

6. Skogman, M. A platform for anti-biofilm assays. Proceedings Pharmaceutical Sciences Department of Biosciences ABO Akademi University Finland, 2012; p. 14 - 63.

7. Naseem S, Khurseed H, Fatimah F, Shaheen S. In vitro evaluation of antimicrobial effect of miswak against common oral pathogens. Pak J Med Sci 2014; 30(2): 398-403.

8. O'toole GA. Microtiter dish biofilm formation assay. J Vis Exp 2011; (47): 2437.

9. Zhang, R, Min C, Yang L, Xiangjun G, Ligeng W. Antibacterial and residual antimicrobial activities against Enterococcus faecalis biofilm: a comparison between EDTA, chlorhexidine, cetrimide, MTAD and QMix. Journal Scientific Reports 2015; 5(12944): 1-5.

10. Li YH, Tian X. Quorum sensing and bacterial interactions in biofilms. Sensors (Basel); 12(3): 2519-2538.

11. Archer NK, Mazaitis MJ, John WC. Staphylococcus aureus biofilms properties, regulation and role in human diseases. Journal Landes Bioscience Virulence 2011; 2(5): 445-59.

12. Gupta A. Biofilm quantification and comparative analysis of MIC and MBIC value for different antibiotic against E.coli. Int Journal Curr Microbiol App Sci India 2015; 4(2): 198-224.

13. Duggan JM, Sedgley CM. Biofilm formation of oral and endodontic Enterococcus faecalis. J Endod 2007; 33(7): 815-8.

14. Mohamed JA, David H. Biofilm formation by Enterococci. Journal of Medical Microbiology 2007; 56: 1581-8.

15. Rezaei A, Glenn O, Virgilio B. Molecular screening of anti-quorum sensing capability of salvadora persica on Enterococcus faecalis. Journal of Hard Tissue Biology 2011; 20(20): 115-24 .

16. Czaczyk K, Myszka K. Biosynthesis of extracellular polymeric substances (EPS) and it's role in microbial biofilm formation. Polish Journal of Environment Studies University of Poznan Poland 2007; 16(6): 799-806

17. Al-Azzawi AJ. The antibacterial effect of herbal alternative, green tea and salvadora persica (siwak) extracts on Enterococcus faecalis. J Bagh College Dentistry 2015; 27(2): 1-4. 